LBNL-39109

\title{
Inversion of Scattered Waves for Material Properties in Fractured Rock
}

\author{
Roland Gritto ${ }^{1,2}$, Valeri A. Korneev ${ }^{1,3}$ and Lane R. Johnson ${ }^{1,2}$ \\ I Center for Computational Seismology, Lawrence Berkeley National Laboratory, Berkeley, \\ California, USA. \\ 2 Seismological Laboratory, University of California, Berkeley, California, USA. \\ 3 Department of Mathematics, Monash University, Clayton, Australia.
}

July, 1999

This research was supported by the Assistant Secretary for Fossil Energy, Division of Oil and Gas of the US Department of Energy under contract DE AC03-76SF00098. Support was also provided by the Airforce Office of Scientific Research under contract F49620-94-I-0197. All computations were carried out at the Center for Computational Seismology of the Lawrence Berkeley National Laboratory. 


\section{DISCLAIMER}

This report was prepared as an account of work sponsored by an agency of the United States Government. Neither the United States Government nor any agency thereof, nor any of their employees, make any warranty, express or implied, or assumes any legal liability or responsibility for the accuracy, completeness, or usefulness of any information, apparatus, product, or process disclosed, or represents that its use would not infringe privately owned rights. Reference herein to any specific commercial product, process, or service by trade name, trademark, manufacturer, or otherwise does not necessarily constitute or imply its endorsement, recommendation, or favoring by the United States Government or any agency thereof. The views and opinions of authors expressed herein do not necessarily state or reflect those of the United States Government or any agency thereof. 


\section{DISCLAIMER}

Portions of this document may be illegible in electronic image products. Images are produced from the best available original document. 


\title{
Inversion of Scattered Waves for Material Properties in Fractured Rock
}

\author{
Roland Gritto ${ }^{1,2}$, Valeri A. Korneev ${ }^{1,3}$ and Lane R. Johnson ${ }^{1,2}$
}

We apply a recently developed low-frequency, non-linear inversion method which includes near and far field terms to a crosshole data set to determine the bulk and shear modulus, as well as the density for a fractured zone in a granitic rock mass. The method uses the scattered elastic wavefield which is extracted from the recorded data before the inversion is performed. The inversion result is appraised by investigating the resolution and standard deviation of the model estimates. The sensitivity of the three parameters to different features of the medium is revealed. While the bulk modulus appears to be sensitive to voids and welded contacts, the density is mostly affected by fractured zones. The shear modulus is least constrained due to the absence of $S$ wave anisotropy information. It is shown that the three medium parameters are generally sensitive to other medium features than those determined by velocity inversions. Thus this method is viewed as a complimentary approach to travel time tomography which provides more insight into the material properties of inhomogeneous media.

\footnotetext{
${ }^{1}$ Center for Computational Seismology, Lawrence Berkeley National Laboratory, Berkeley, California, USA

${ }^{2}$ Seismological Laboratory, University of California, Berkeley, California, USA.

${ }^{3}$ Department of Mathematics, Monash University, Clayton, Australia.
} 


\section{Introduction}

The inversion of scattered elastic waves to determine subsurface structures has become an active area of research in recent years. The need to determine medium properties like elastic moduli, density, scattering and anelastic attenuation (Scherbaum, 1990; Lees and Lindey, 1994), to detect and locate fracture zones (Vasco et al. 1996), partially molten zones (Zucca et al., 1987; Romero et al., 1993) and hydrocarbon deposits, for example, has caused the development of waveform inversion in addition to travel time tomography techniques.

Elastic waves propagating through an inhomogeneous medium are affected in two ways. Their travel time is delayed or advanced depending on the nature of the inhomogeneities (Peterson, 1986; Nolet, 1987), and energy from the incident wave is scattered throughout the medium, arriving at different times at the point of observation. The bulk of this scattered energy is delayed and becomes evident as various phases arriving after the direct wave in the seismogram (Aki, 1969; Korn, 1990). This energy is generally referred to as coda. However, the coda contains valuable information about the medium and is the target of waveform inversion techniques (Tura, 1990).

In recent years the demand to use seismic waves as a diagnostic tool to estimate high resolution models of the subsurface and to extract a variety of physical parameters which are needed to model subsurface processes has increased. Waveform inversion techniques that utilize amplitude information appear to be suitable approaches for estimation of these parameters. But most of these waveform approaches solve for velocities, rather than physical parameters like elastic moduli and density (Wu et al., 1987, Lo et al., 1988; Tura et al., 1992). In principle, the velocity information can provide some insight into elastic properties, in cases where both $\mathrm{P}$ and $\mathrm{S}$ waves have been determined separately. However, without additional measurements (e.g density) they fail to provide independent information about the physical parameters of the medium. Furthermore, the material parameters may cancel or reduce their effect in the equations for the velocities (Gritto et al., 1995b), and thus they may not be suited to provide the true values of the physical parameters of the medium.

Most of the high resolution seismic surveys are cross hole experiments, with the objective of estimating the physical parameters between the boreholes.
Of particular interest are regions close to the wells, as the parameters can be estimated by complimentary measurements in the boreholes, thus providing constrains for the inversion. However, it has been pointed out by Gritto et al. (1995a) that scattered amplitudes in the near field of inhomogeneities can be up to 3 orders of magnitudes larger than in the far field. Therefore, it is important to include near field terms in the inversion to provide reliable estimates of the parameters in the vicinity of the wells. Furthermore, most waveform inversions rely on linearized methods based on weak scattering assumptions which are difficult to justify in many heterogeneous media. As stated above, the possible canceling effect of the material parameters in the equation of the velocities may hide the fact that their deviation from background values may be stronger than suggested by the velocities. Therefore, in order to estimate medium parameters, a non-linear approach may be required for most heterogeneous media.

A method recently developed by Gritto et al. (1996) addresses most of the points mentioned above. It is a non-linear 3-dimensional inversion method for low frequency scattered elastic waves that can account for anelastic attenuation. The solution contains near and far field terms for the incident and the scattered waves which allows for an inversion in the vicinity and farther away from the source and receiver wells. Furthermore it solves for the bulk and shear modulus and the density in an exact non-linear way, thus avoiding less reliable iterative schemes. In the following this method will be applied to a cross well experiment at the Fracture Zone Investigation (FRI) site of the Grimsel Rock Laboratory, Switzerland, to investigate whether it is possible to detect, delineate, and estimate the material properties of a fracture zone. Furthermore, it will be investigated whether it is possible to treat large inhomogeneities by a series of point scatterers under the assumption of single scattering.

\section{Method}

In this study we follow the mathematical development of Gritto et al. (1996). The scattering process is based on the solution describing the scattered elastic wavefield for the incidence of a spherical $P$ wave onto an inhomogeneity of variable shape but approximately equal dimensions. The solution is a low frequency approximation to the exact solution derived for the scattering by a sphere (Korneev and Johnson, 1993). In this derivation single scattering is assumed. 
Furthermore, the wavelength of the incident wave is assumed to be larger than the dimensions of the inhomogeneity. The inherently non-linear problem can be solved directly by introducing new parameters, referred to as moments, which are related to the elastic parameters by equations that have analytic solutions. Thus by inverting the scattered elastic waves for the moments first, it is possible to subsequently solve for the bulk and shear modulus, as well as for the density in an exact direct way (Gritto, 1996). The advantage of solving for the moments first, beyond the obvious improvement of being able to invert for arbitrarily large contrasts.in a direct way, lies in the fact that the moments are dimensionless properties which helps to stabilize the inversion. In contrast the elastic moduli and the density differ by 6 orders of magnitude, which may cause instabilities even for well conditioned problems.

In the following paragraph the concept of deriving the inverse problem using matrix notation will be considered so as to provide a basis for the definition of the model resolution as applied in the last section of this study. The parametrization of the model into voxels of equal volume, allows the problem to be expressed in matrix form as

$$
\mathrm{U}=\mathbf{C} \mathbf{M}
$$

where $\mathrm{U}$ is the data vector consisting of scattered $\mathrm{P}$ and $S$ waves, $M$ is the model vector containing the unknown moments for each voxel, and $\mathbf{C}$ is a matrix which describes the physics of the scattering problem. It connects data and model parameters through the Green function for the scattering process. Thus, upon computing the inverse of $\mathbf{C}$ the model parameters can be determined as

$$
\mathbf{M}=\mathbf{C}^{-1} \mathbf{U}
$$

The method chosen to compute the inverse of $\mathbf{C}$ is singular value decomposition (SVD). Although this is a time consuming method requiring large computational resources, it provides good diagnostic insight into the inversion process. SVD produces a decomposition of the original matrix $\mathbf{C}$ into the product of three matrices (Menke, 1989).

$$
\mathbf{C}=\mathbf{W} \boldsymbol{\Lambda} \mathbf{V}^{\mathbf{T}}
$$

where $W$ is a matrix of orthogonal eigenvectors spaning the data space, while similarly $\mathbf{V}$ is a matrix of eigenvectors that spans the model space. The matrix $\boldsymbol{\Lambda}$ is the diagonal matrix of non negative elements called singular values. The singular values are arranged in decreasing order with the possibility of zero values. However, before the inverse can be computed the zero singular values and the related vectors in $W$ and $V$ have to be eliminated from the system. Thus the rank of the matrix is reduced which is indicated by the index $\mathrm{p}$ denoting the number of non-zero singular values. Once the decomposition is obtained, the inverse of $\mathbf{C}$ is readily given by

$$
C^{-1}=V_{p} \Lambda_{p}^{-1} W_{p}^{T}
$$

and thus, the solution to the inverse problem is given by

$$
\widehat{\mathrm{M}}=\mathrm{V}_{\mathrm{p}} \Lambda_{\mathrm{p}}^{-1} \mathrm{~W}_{\mathrm{p}}^{\mathrm{T}} \mathrm{U}
$$

where $\widehat{\mathbf{M}}$ denotes the estimate of $\mathbf{M}$. Furthermore, the decomposition of $\mathbf{C}$ into the matrices $W_{p}$ and $V_{p}$ allows the performance of the inversion to be investigated. A measure of the resolving power of the model parameters can be computed as

$$
\mathbf{R}=\mathbf{V}_{\mathbf{p}} \mathbf{V}_{\mathbf{p}}^{\mathbf{T}}
$$

In a perfectly resolved model, $\mathbf{R}$ is equal to the identity matrix $I$. If model parameters become dependent on each other and can no longer be resolved, the value of the diagonal decreases and spreads out to neighboring elements. In this sense the rows of $\mathbf{R}$ are a qualitative measure for the resolution of each model parameter. In the last section the model resolution will be computed to appraise the inversion result by determining whether independent model estimates are obtained.

\section{Geology and Data Acquisition}

The United States Department of Energy (DOE) and the Swiss Cooperation for the Storage of Nuclear Waste (NAGRA) conducted several experiments to investigate the effect of fractures on the storage of 
nuclear waste in underground repositories. The experiment was carried out at the Grimsel test site in Switzerland, with the primary goal to determine the nature of wave propagation in fractured rocks and to relate seismological to hydrological parameters. The experiment described here was carried out at the FRI site with the intention to delineate and determine the material parameters of a known fracture zone in a highly foliated granitic host rock. The geometry of the FRI site is shown in Figure 1. Two main service tunnels provide access to a zone of fracturing striking NE-SW. The zone appears to be an area with varying concentration of thin fractures, determined from cores taken from two horizontal boreholes (BOFR 87.001 and BOFR 87.002) as indicated by the short lines along the holes in Figure 1. In addition to the mapped fractures, a change in color of the granodiorite may indicate an additional feature in the upper half of the panel, indicated by the dashed line. The data were collected between the two boreholes BOFR 87.001 and BOFR 87.002 each of $20 \mathrm{~m}$ length, with 39 sources located in the first and 39 receivers in the second hole, respectively, separated by a distance of 10 $m$. During the experiment, the horizontal holes were water filled to improve the source and receiver coupling. Additionally, the receiver, a 3-component geophone, was clamped to the borehole wall. Although the setup reveals a typical 2-dimensional crosswell geometry, the fracture zone can be expected to extend in both directions perpendicular to the plane.

\section{Data Processing}

Figure 2 shows a typical source gather for a source located at $z=1.35 \mathrm{~m}$ in borehole BOFR 87.001 . The reverberative nature of the incident wave is apparent behind the first arrival and is caused by multiple reflection in the water filled boreholes. Several faint arrivals, representing reflections off tunnel walls, $\mathrm{S}$ waves radiated at an angle of $45^{\circ}$ by the source, and tube waves appear in the section after $t=5.5 \mathrm{~ms}$ (Tura et al., 1992).

The theory of the adopted inversion approach is formulated for the scattered wavefield only, which requires the elimination of the incident wavefield from the recorded data. However, the reverberations in the present example make it difficult to correctly estimate the incident wave and remove it from the data. In addition to the incident field, the data are contaminated by the radiation pattern of the source, the source and receiver coupling to the borehole, and anelastic atten- uation in the medium. Corrections for these effects should be applied before an inversion is attempted. The correction for anelastic attenuation is only performed for the incident wave so that its correct waveform can be determined. Although the inversion algorithm is set up to solve for anelastic attenuation, this goal was not pursued in our study because of computational limitations. Therefore, after removal of the incident wave, the scattered field was not corrected for anelastic attenuation.

To correct for directional differences in energy radiation at the source, a cosine operator $(\cos \Theta)$ is applied, where $\Theta$ is the angle between the horizontal and the source receiver direction. This operator was found by estimating the amplitude of the first arrival (after correcting for geometrical spreading and preliminary anelastic attenuation) while the receivers were moved in a crosshole fashion around a fixed source in a homogeneous region of the host rock. The cosine function was found to best fit the amplitude pattern (Majer et al., 1990). In a similar experiment, the $P$ wave velocity was determined as a function of direction of propagation. The granitic rock shows a high level of foliation, parallel to the strike of the fracture zone. This foliation produces $\mathrm{P}$ wave anisotropy as large as $8 \%$, with the symmetry axis normal to the plane of fracturing (transverse isotropy). Hence, the fast direction is parallel, and the slow direction perpendicular to the strike of the fracture zone. In general, the $P$ wave anisotropy may be approximately represented by (Backus, 1965)

$V_{p}^{2}=A+B \sin (2 \Phi)+C \cos (2 \Phi)+D \sin (4 \Phi)+E \cos (4 \Phi)$

where $\Phi$ is the angle of propagation with respect to the horizontal, and the coefficients were determined by Majer et al. (1990) as

\begin{tabular}{|l||c|c|c|c|c|}
\hline coeff. & $\mathrm{A}$ & $\mathrm{B}$ & $\mathrm{C}$ & $\mathrm{D}$ & $\mathrm{E}$ \\
\hline value $[\mathrm{m} / \mathrm{s}]^{2}$ & 27.942 & 1.375 & -0.633 & -0.309 & -0.185 \\
\hline
\end{tabular}

Although no anisotropy estimation was performed for the $S$ wave velocity, the background value was determined at $V_{s}=3200 \mathrm{~m} / \mathrm{s}$. It is expected, however, that the host rock contains a significant degree of $S$ wave anisotropy, and disregarding this effect will reduce the quality of the inversion results, as a considerable amount of scattered energy is converted into $S$ waves. 
To estimate the mean anelastic background attenuation $\alpha$ and the factors governing the source and receiver coupling at their locations in the boreholes, the amplitudes of the first arrivals were measured and the parameters estimated in a least squares sense. The coupling factors and attenuation have to be determined simultaneously, as they have similar effects on the amplitude of the wavefield, and an inversion of a single parameter is not possible without estimating the others first. The wavefield is corrected for geometrical spreading and the radiation pattern of the source. Subsequently, the traces are aligned along their arrival times and the amplitudes of the first minima determined. A total of 1521 amplitude values were inverted to solve for the 79 unknowns (78 source and receiver coupling factors and the average background attenuation $\alpha$ ). The background attenuation is estimated to be $\alpha=0.36$ neper $/ \mathrm{m}$. Assuming a linear frequency dependence for $\alpha$, a background velocity of $V_{p}=5270 \mathrm{~m} / \mathrm{s}$, and a peak frequency dominating the incident pulse of $f_{0}=10 \mathrm{KHz}$, the relation $Q=\pi f / \alpha V_{p}$ produces a frequency independent quality factor of $Q=17$. The value for $\alpha$, although it seems to be low, is not unreasonable for this highly foliated medium considering the short wavelength of $0.53 \mathrm{~m}$ of the incident pulse. It is representative of the mean background attenuation, however, and does not take into account local areas of abnormal attenuation.

The results for the normalized source and receiver coupling factors are presented in Figure 3 . It can be seen that the source factors vary around the value of 1.0 with larger deviations between source position 10 to 20 , corresponding to $z=5 m-10 m$, respectively. Referring to Figure 1 it becomes obvious that this is the range where the assumed fracture zone intersects borehole BOFR 87.001. As the factors are larger than unity towards the edges but less than unity in the center of the fracture zone, the physical parameters may be varying across the fault area, indicating that the fracture zone may consist of an accumulation of thin parallel cracks. The trend for the receiver factors differs from the source factors but is generally consistent with the geology in the receiver borehole. A monotonic increase between values of 0.5 to 1.0 between receiver position $1(z=1.5 \mathrm{~m})$ and $15(z=8.5 \mathrm{~m})$ is evident in Figure 3b. The low values are too small to be explained by borehole effects only, since the holes were carefully drilled and cored to determine the status of the medium. Since the inversion simultaneously solves for the mean value of $\alpha$, but does not recover local attenuation anomalies, such anomalies will have an effect on the wavefield, particularly if these zones are close to certain receivers. Such an anomalous attenuation zone was detected by Majer et al. (1990) for a region close to borehole BOFR 87.002 between the receiver position $1(z=1.5 \mathrm{~m})$ and $10(z=6.0 \mathrm{~m})$. This result is mirrored by the low values in Figure 3b. Figure 1 shows borehole BOFR 87.002 intersecting the fracture zone at approximately $(z=16.0 \mathrm{~m})$ (receiver position 32). Again, the receiver factors reveal a maximum, before they drop to lower values towards the center of the fracture zone at the end of the borehole. Thus, the source and receiver coupling factors seem to relate to the geological features, indicating a non uniform fractured zone and possibly a high attenuation area in the SE corner of the study area.

After correcting the total wavefield for the source and receiver coupling factors and the background attenuation, the first arrivals should be a good representation of the source wavelet. Therefore, for every source position, the traces are stacked to produce a representative source signal for each source location. Finally, the 39 source wavelets are stacked to produce the common source signal representative of all source positions. This wavelet is shown in Figure 4. The reverberative nature is evident after the first impulse for almost $0.6 \mathrm{~ms}$. In contrast to many traces, the amplitudes are not constant but appear to be attenuated after the first pulse. The reverberations are caused in part by multiple reflections in the receiver borehole (Tura, 1990). However, in contrast to the receiver, the source was not clamped in the borehole, as the water was intended as the coupling medium to the borehole wall. Thus, after the first source pulse, a fraction of the energy reverberates in the source hole and each reverberation radiates energy into the formation. Therefore, the multiples are likely to be a mixture of source and borehole reverberations. It will be discussed in a later section what length of the source wavelet provides the best deconvolution operator for the scattered wavefield.

The first $0.6 m s$ (150 samples) of the source wavelet are convolved with a 3-d Green function to compute a representative incident field which should be subtracted from the total field. However, because of the large amplitudes of the reverberations in many single traces which did not stack constructively to equally large amplitudes in the source wavelet, it was not possible to remove the incident field and the reverberations effectively. Without the removal of the reverberations, however, the inversion will not produce re- 
liable results as they produce the largest amplitudes in the seismogram after the removal of the incident field. Therefore, the incident field and the reverberations are suppressed by applying a one sided Hamming window to the beginning of the traces to damp an interval of $0.6 \mathrm{~ms}$ after the first arrival time. The window length was estimated from the source wavelet in Figure 4, where the reverberations appear to be present up to $0.6 \mathrm{~ms}$. The windowing process simultaneously damped all scattered phases that coincided approximately with the incident field in the seismogram. These phases which appear with little travel time separation from the incident field in the seismogram are scattered in the vicinity of the sources or the receivers (near field scattered phases). To avoid incident $S$ waves and tube waves in the coda of the traces, visible after $5.5 \mathrm{~ms}$ in Figure 2, the end of the traces are damped, again using a one sided Hamming window. The muted wavefield representing the bulk of the scattered energy is shown in Figure 5, where the geometry of sources and receivers is the same as for Figure 2. The windowing process limits the scattered phases primarily to forward scattered energy.

In a final step before inversion, the scattered field has to be deconvolved by the source wavelet to normalize the amplitudes by the source strength. In the last paragraph, it was argued that part of the reverberative nature of the source wavelet is due to reverberations in the source borehole. In this case the borehole source radiates energy into the formation with every reverberation. This delayed energy has to be taken into account for a successful deconvolution by the source signal. For this reason the length of the deconvolution operator is chosen to be $0.6 \mathrm{~ms}$, after which the reverberations of the wavelet are sufficiently damped. After deconvolution, the spectral values of the scattered field are taken as input for the inversion algorithm.

\section{Data Inversion}

The inverse problem can be solved in the frequency domain for any specific frequency. The images of the inversion results are compiled by stacking 13 individual inversion results, each computed for one single frequency ranging from $3051 \mathrm{~Hz}$ to $5981 \mathrm{~Hz}$ with an interval of $244 \mathrm{~Hz}$. Assuming a background $\mathrm{P}$ wave velocity of $V_{p}=5270 \mathrm{~m} / \mathrm{s}$, this translates to a wavelength of $\lambda_{p}=1.7 \mathrm{~m}$ and $\lambda_{p}=0.9 \mathrm{~m}$ for the incident wave, respectively. Unsing a voxel length of $1 m$, the wavelengths vary from the Mie scattering range $\left(\lambda_{p}=0.9 m\right)$ to a value $\left(\lambda_{p}=1.7 m\right)$ that lies between the ranges of Mie and Rayleigh scattering. The inversion was performed using lower frequencies as well, but the resulting images did not reveal sufficient resolution and therefore were dismissed from the final stack.

Figure 6 reveals the geometry of the area which is parametrized in the model. As indicated in Figure 2 the boreholes are slightly slanted with respect to the z-axis of the coordinate system. An area $9 m \times$ $19 \mathrm{~m}$ was parametrized into 171 voxels of $1 \mathrm{~m}^{3}$ each. Data from all 39 source and receiver positions are used. After the inversion, each panel is smoothed applying a cubic b-spline interpolation of Michelini et al. (1991) before generating the final plot. The background values of the inversion are determined using the $V_{p}$ and $V_{s}$ values given above and a mean density of $\rho=2650 \mathrm{~kg} / \mathrm{m}^{3}$ (Majer et al., 1990). This translates into a bulk and shear modulus of $32.42 G P a$ and 27.14GPa, respectively. However, to generalize the inversion results the images present the anomalies in per cent units relative to the background values.

The inversion results in Figure 6 were obtained by taking the $P$ wave anisotropy into account but neglecting the correction for anelastic attenuation. In general this will cause the scattered amplitudes to be more attenuated, but zones of higher attenuation could also be a source of increased scattering. The image of the bulk modulus (Figure 6a) reveals several features that can be related to the inferred geological interpretation as suggested in Figure 1. In the bottom half of the image a crossing pattern of low bulk modulus anomalies can be seen. The suggested fracture zone is not clearly visible due to a more pronounced feature running from the lower left corner to borehole BOFR 87.002 (No. 1 in Figure 6a). Referring to Figure 1 this could be the effect of the borehole labeled CO 1 connecting the AU Tunnel with borehole BOFR 87.002. A possible explanation may be provided by the nature of the fracture zone. In contrast to a clear fracture, this zone probably consist of several thin parallel fractures with a more gradual reduction in the elastic parameters, and thus it may not reveal such a strong contrast. The borehole, however, provides a sharp contrast to the background medium and therefore constitutes a strong inhomogeneity for elastic waves. However, it cannot be expected that values close to $-100 \%$ will be obtained for the bulk modulus in the vicinity of the borehole region, as the inversion solves for the product of volume times parameter deviation. Because the selected volume is 
$1 \mathrm{~m}^{3}$ per voxel, the negative perturbation of the bulk modulus for the small borehole have to be averaged over $1 \mathrm{~m}^{3}$, which will decrease the absolute value of the deviation from the background. A similar crossing feature of low values in bulk modulus is visible in the upper half of the panel (No. 2 in Figure 6a). It coincides with a low velocity anomaly found by Majer et al. (1990) and Vasco et al. (1996) which runs diagonally across the upper half of the survey area. An additional anomaly is visible striking from BOFR 87.002 upward towards the source hole (No. 3 in Figure 6a). The interpretation could be a possible suture zone between two differently colored host rocks in the area (dashed line in Figure 1). An explanation could be based on the fact that this welded contact constitutes a better scatterer than the fracture zone and thus produces a more pronounced feature. Side effects from the tunnel walls are not apparent, which is due to the windowing at the end of the traces. The large positive anomalies in the vicinity of the receiver hole BOFR 87.002 lie in a zone of large uncertainty as discussed below. energy. These areas lie in the near field of the receivers, and therefore, the associated scattered waves were muted out during the removal of the incident wave. The results for the shear modulus partly support this interpretation, although they reveal the smallest perturbation of the three parameters. The suggested suture zone may be present as a weak structure below its position in the upper panel (No. 1 in Figure 6b). However, $S$ wave anisotropy is not accounted for in the inversion, and therefore the location of the shear modulus anomalies may be shifted relative to the other parameters. The location of the bulk modulus should be well constrained as it is affected by the $P$ waves only, whereas the density, being constrained by $P$ and $S$ waves, may not be as much affected by deviations in the $S$ wave velocity as the shear modulus. The right panel, displaying the density data, shows a strong anomaly runing from the upper right corner towards the middle of the panel (No. 1 in Figure 6c). Again its location coincides with the low velocity anomaly as determined by Majer et al. (1990) and Vasco et al. (1996). The presence of a possible fracture zone is supported by a small set of fractures found at the western end of borehole BOFR 87.002, as indicated by the short lines in Figure 1. However, later on we will show that this density anomaly carries large uncertainties. The suture zone may be indicated by a faint anomaly in the upper half of the panel (No. 2 in Figure 6c), while the fracture zone in the bottom half is not well constrained by the density.

The results of the three parameters clearly show the sensitivity to changes in material properties contained in the process of elastic wave scattering. Because of the limitations in the data processing, the relative amplitudes of the moduli are the least constrained parameters in the inversion. Similarly, the consideration of $\mathbf{S}$ wave anisotropy should improve the location of the anomalies of the shear modulus. However, despite these constrains it is evident that the three parameters are affected in distinct ways by the encountered geological features. It appears that the bulk modulus is more sensitive to drastic changes (e.g. borehole or welded contact) than it is to gradual changes in a zone consisting of several fractures. The density on the other hand may be sensitive to both type of features, whereas no real conclusion can be drawn for the shear modulus from these results. However, this suggests as indicated before (Gritto et al. $1995 \mathrm{~b}$ ), that the difference in response of the three parameters may lead to decreased effects in the seismic velocities.

To demonstrate this point, the $\mathrm{P}$ and $\mathrm{S}$ wave velocities as well as the $V_{s} / V_{p}$ ratio are computed for the inversion results of Figure 6 and presented in Figure 7. As can be seen, the same parameters produce different features when displayed in terms of the velocities. The $P$ and $S$ wave velocities suggest the presence of a low velocity anomaly in the lower right corner of the panel (No. 1 in Figure 7), possibly indicating the fracture zone, which is known to intersect the eastern tunnel (AU Tunnel in Figure 1) at $(x=8 m, z=20 m)$. Contrary to the bulk modulus the presence of borehole CO 1 is not as evident in either the $P$ or $S$ wave velocity. The suture zone in the upper half is not visible, whereas the anomaly crossing from the upper right corner towards the middle of the panel (No. 2 in Figure 7a,b) is evident as a high velocity anomaly, due to the low values in density for the same location in Figure 6. Because these features are similar in $P$ and $S$ wave velocities, they do not produce anomalies in the $V_{s} / V_{p}$ ratio (Figure $7 \mathrm{c}$ ). The most apparent feature in the $V_{s} / V_{p}$ ratio is what appears to be a local anomaly in the center region of borehole CO 1 (No. 1 in Figure 7c). A comparison with Figure 6a reveals that this anomaly is also apparent in the bulk modulus. As this is the region where the fracture zone intersects the borehole as indicated in Figure 1, a possible interpretation of this small but persistent anomaly could be a breakout zone in the borehole. However it remains to be 
seen whether this interpretation can be confirmed by caliper logs that may have been taken in borehole $\mathrm{CO}$ 1. In general, it is evident that the elastic moduli and the density respond distinctively to different types of geological features and are useful in extracting fundamental information which can be displayed in various combinations to provide a more profound understanding of heterogeneous subsurface structures.

The inversion problem considered above is overdetermined since all 39 source and receiver positions are used and a total of 513 model parameters are inverted. Although the inversion was damped to reduce the effects of small singular values, the model parameters are reasonably well resolved. Figure 8 reveals the diagonal values of the resolution matrix as derived in equation (6). A value of 1 for a certain parameter in a voxel indicates that this estimate is independent of neighboring parameters. Deviations from unity denote an increase in the degree of dependence between model parameters. It can be seen in Figure 8 that the least resolved parameter is the bulk modulus with values as low as 0.3 along the edges of the model near the access tunnels (Figure 8a), while most of its values remain above 0.5 . In contrast, the values for the shear modulus and the density lie above 0.6 throughout the parameter space. The drop in model resolution for the bulk modulus is caused by the fact that it is only constrained by the scattered $P$ waves, whereas shear modulus and density are determined by both $P$ and $S$ waves. It is evident that the edges along the source and receiver boreholes are best resolved (up to 0.95) whereas the central regions reveal the lowest values. This drop in the center relates to the less perfect angular coverage, where the waves are more likely to sample a voxel in straight transmission (Gritto, 1995 c). Concerning the model resolution, it can be stated that most of the features in Figure 6 are resolved and to a certain degree independent of each other.

The decomposition of the matrix system allows the standard deviation for the model estimates to be computed. However, a prerequisite is that the covariance matrix of the data is known which is not the case in the present problem, nor is it possible to reliably estimate this uncertainty, given the lack of information on data aquisition procedures and various simplifying assumptions that have been made. Therefore, the standard deviation is estimated from the variance of the 13 individual inversion results that are the basis for the image in Figure 6. Figure 9 shows the standard deviation of the images expressed in per cent units based on the background values. Thus the values are directly comparable to the anomalies in Figure 6. It is evident that the standard deviation increases for large estimates, since it reaches maximum values of $30 \%$ for the bulk modulus which revealed the largest anomalies in Figure 6. However, it can be stated that the anomalies discussed in Figure 6a appear to be real, as the standard deviation for the associated areas reveals values below $13 \%$, thus remaining below $65 \%$ of the model estimate. In contrast, the density anomaly in Figure 6c seems questionable as the standard deviation reaches values of $\sim 6 \%$ (Figure 9c) that are larger than the model estimate of $\sim-5.5 \%$ in Figure $6 c$.

As stated above, because of the lack of information on $S$ wave velocity as a function of azimuth, the inversion was performed taking $P$ wave anisotropy only into account. It should be noted that $S$ wave anisotropy can be as easily implemented in the inversion process as the $\mathrm{P}$ wave anisotropy presented here. However, in the present example, the effect of scattered $S$ waves may have been additionally suppressed by the windowing at the end of the traces, which is supported by the weak anomalies in the shear modulus. An additional cause for these weak anomalies may be the anelastic attenuation of the medium. Because the wavefield is not corrected for this parameter and as the inversion is done one frequency at a time, the attenuating effect will be stronger for the $S$ waves as their wavelengths are shorter and therefore, they are more strongly attenuated than $P$ waves over the same travel distance assuming comparable $Q$ values.

\section{Conclusions}

The application of inverting scattered waves to determine subsurface material parameters revealed the following results. Before a successful inversion of the scattered wavefield can be attempted, it is necessary to remove the incident wave. However, because of strong reverberations in the source and receiver hole the incident field could not be modeled accurately enough to be subtracted, and thus had to be removed from the traces. The muting affected the scattered waves by partially removing near field and $S$ wave scattered energy.

However, the inversion of the scattered wavefield produced reasonable results that can be related to the geology as determined from the tunnel and borehole walls. It appears that the two elastic moduli and the density respond to different features in different ways, and therefore may indicate a good method to 
distinguish between e.g. an open fracture zone and a closed welded fracture. Furthermore, the commonly used images of $V_{p}$ and $V_{s}$ velocities and their ratio can be easily computed to allow other tomographic interpretations.

The fracture zone is embedded in a relatively homogeneous granite and it can be expected that it extends to both sides perpendicular to the experimental plane. Thus out of plane scattered amplitudes will be evident in the seismogram traces, which will contribute to an overestimation of the parameters to be determined in the plane. A possible test would be to invert for a 3-dimensional medium e.g. consisting of 3 planes parallel to each other extending in the $y$-direction. Thus out of plane scattering could be traced back to its origin and the image could be improved. However, sufficient source and receiver coverage is necessary which was not the case for this experiment.

The inversion result for the bulk modulus suggests that it is possible to model a spatially extensive anomaly by a series of point scatterers. The lack of $S$ wave anisotropy data severely limited the results for the shear modulus and the density. This indicates that a good estimate of the background medium parameters is essential before a successful inversion can be attempted. Therefore, the presented method should be seen as a complimentary tool to transmission tomography, where the results of the velocity analysis can be incorporated in a starting model that will allow the determination of heterogeneous properties or large contrasts of the medium which are elusive to travel time tomography approaches.

Acknowledgments This research was supported by the Assistant Secretary for Fossil Energy, Division of Oil and Gas of the US Department of Energy under contract DE AC03-76SF00098. Support was also provided by the Airforce Office of Scientific Research under contract F49620-94-I-0197. All computations were carried out at the Center for Computational Seismology of the Lawrence Berkeley National Laboratory.

\section{References}

Aki, K., Analysis of the seismic coda of local earthquakes as scattered waves, J. Geophys. Res., 74, 615-631, 1969.

Backus, G., Possible forms of seismic anisotropy in the uppermost mantle under oceans, Journal of Geophysical Research, 70, 3429-3439, 1965.

Gritto R., Korneev V. A., and Johnson L. R., Lowfrequency elastic-wave scattering by an inclusion: limits of applications, Geophys. J. Int., 120677 692,1995 a.

Gritto R., Kaelin B., and Johnson L. R., Analysis of crustal heterogeneity with application to wave propagation at the KTB site, Earth Sciences Division, Lawrence Berkeley National Laboratory, 1995 b.

Gritto R., Rayleigh scattering and nonlinear inversion of elastic waves, Ph.D. Thesis, University of California at Berkeley, $1995 \mathrm{c}$.

Gritto R., Korneev V. A., and Johnson L. R., Nonlinear 3-dimensional inversion of low frequency scattered elastic waves. Geophys. J. Int., submitted, 1996.

Korn, M., A modified energy flux model for lithospheric scattering of teleseismic body waves, Geophys. J. Int., 102, 165-175, 1990.

Korneev, V. A., and Johnson, L. R., 1993, Scattering of elastic waves by a spherical inclusion - 2. Limitation of asymptotic solutions, Geophys. J. Int., 115, 251-263, 1993.

Lees, J. M. and Lindey, G. T., Three-dimensional attenuation tomography at Loma Prieta: inversion of $t^{*}$ for $Q$, in Long Valley caldera, California, $J$. Geophys. Res., 99, 6843-6863, 1994.

Lo, T. W., Toksöz, M. N., Xu S. H., and Wu R. S., Ultrasonic laboratory tests of geophysical tomographic reconstruction, Geophysics, 53, 947-956, 1988.

Majer, E. L., Myer, L. R., Peterson, J. E., Karasaki, K., Long, J. C. S., Martel, S. J., Blümling P., and Vomvoris, S., Joint seismic, hyrological, and geomechanical investigations of a fracture zone in the Grimsel rock laboratory, Switzerland, NAGRADOE Cooperative Project Report, 1990.

Menke W., Geophysical Data Analysis: Discrete Inverse Theory, Academic Press, Inc., 1989. 
Nolet, G. (ed.), S\&EG Seismic Tomography, D. Reidel Publishing Company, 1987.

Peterson, J. E., The application of algebraic reconstruction techniques to geophysical problems, $P h . D$. Thesis, University of California at Berkeley, 1986.

Romero A. E. Jr., McEvilly T. V., Majer E. L. and Michelini A., Velocity structure of the Long Valley caldera from the inversion of local earthquake $P$ and $\mathrm{S}$ travel times, J. Geophys. Res., 98, 1986919879, 1993.

Scherbaum, F., Combined inversion for the threedimensional Q structure and source parameters using microearthquake spectra, J. Geophys. Res., 95, 12423-12438, 1990.

Tura, M. A. C., Acoustic and elastic diffraction tomography and its application to fracture detection, Ph.D. Thesis, University of California at Berkeley, 1990.

Tura, M. A. C., Johnson L. R., Major E. L. and Peterson J. E. Jr., Application of diffraction tomography to fracture detection, Geophysics, 57, 245-257, 1992.

Vasco, D. W., Peterson J. E. Jr. and Major E. L., A simultaneous inversion of seismic travel time and amplitudes for velocity and attenuation, Geophysics, in press, 1996.

Wu, R. and Toksöz, M. N., Diffraction tomography and multisource holography applied to seismic imaging, Geophysics, 52, 11-25, 1987.

Zucca J. J., Kasameyer, P. W. and Mills J. M. Jr., Observation of a reflection from the base of a magma chamber in Long Valley Caldera, California, Bull. Seis. Soc. Am., 77, 1674-1687, 1987.
Figure 1. Experimental design and geology at the Grimsel FRI site. The sources were placed in hole BOFR 87.001, while the receivers were located in hole BOFR 87.002.

Figure 2. Source gather of total wavefield for source location $z=1.35 \mathrm{~m}, \mathrm{a}$ ) $\mathrm{x}$-component, b) z-component.

Figure 3. Normalized source and receiver coupling factors, a) source factors, b) receiver factors.

Figure 4. Representative source wavelet averaged over all source locations.

Figure 5. Source gather of scattered wavefield for source location at $z=1.35 \mathrm{~m}$, a) x-component, b) zcomponent.

Figure 6. Inversion result for the material parameters. Stars denote sources, while receivers are indicated by triangles. The result represents a stack of 13 individual frequencies, ranging from $3051 \mathrm{~Hz}$ to $5981 \mathrm{~Hz}$, a) bulk modulus, b) shear modulus, c) density.

Figure 7. Velocity and $V_{s} / V_{p}$-ratio maps determined from the inversion results of Figure 6 , a) $\mathrm{P}$ wave velocity, b) $\mathrm{S}$ wave velocity, c) $V_{s} / V_{p}$-ratio.

Figure 8. Model resolution for the parameters in Figure 6, a) resolution for bulk modulus, b) resolution for shear modulus, $c$ ) resolution for density.

Figure 9. Standard deviation (sd) for the model estimates in Figure 6, a) sd for bulk modulus, b) sd for shear modulus, c) sd for density.
This preprint was prepared with the AGU IATEX macros v3.0. File grimsel formatted 1999 July 27. 


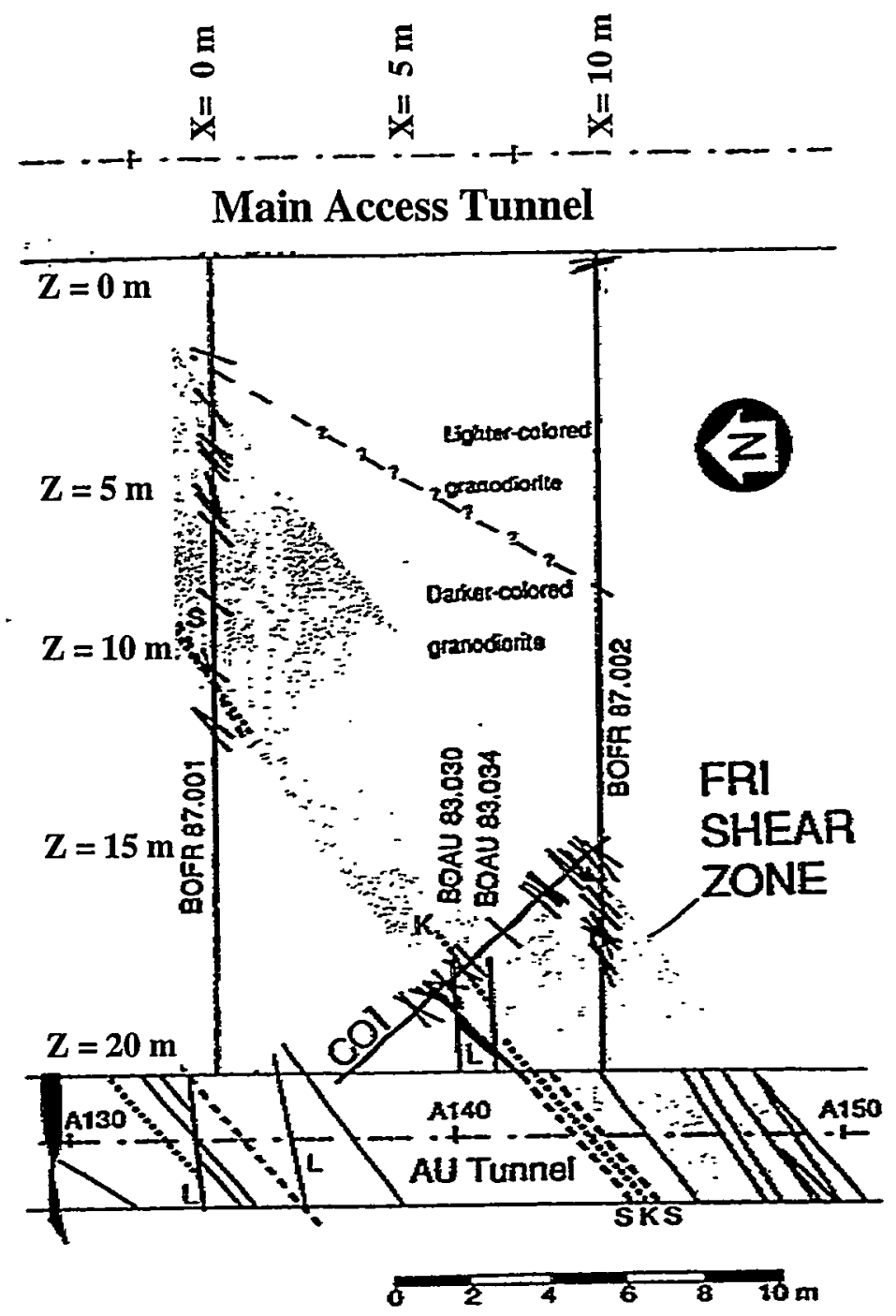

Figure 1 
N
을
은
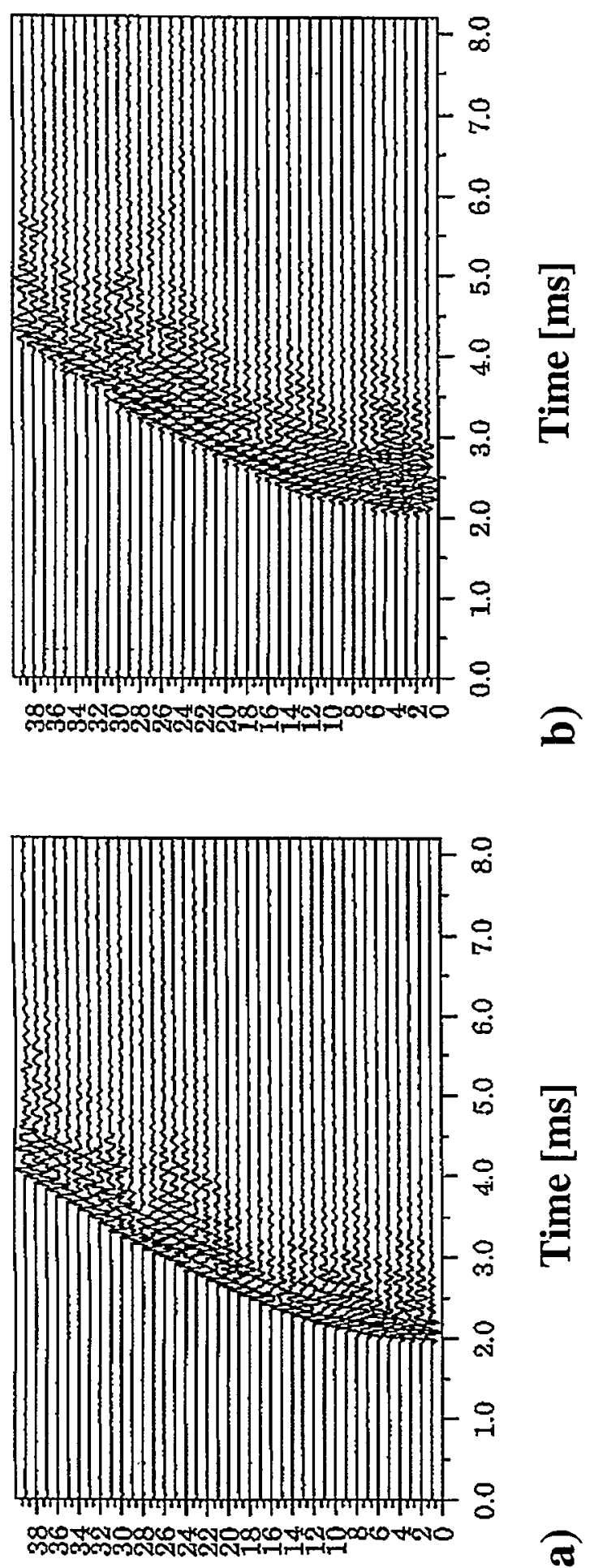

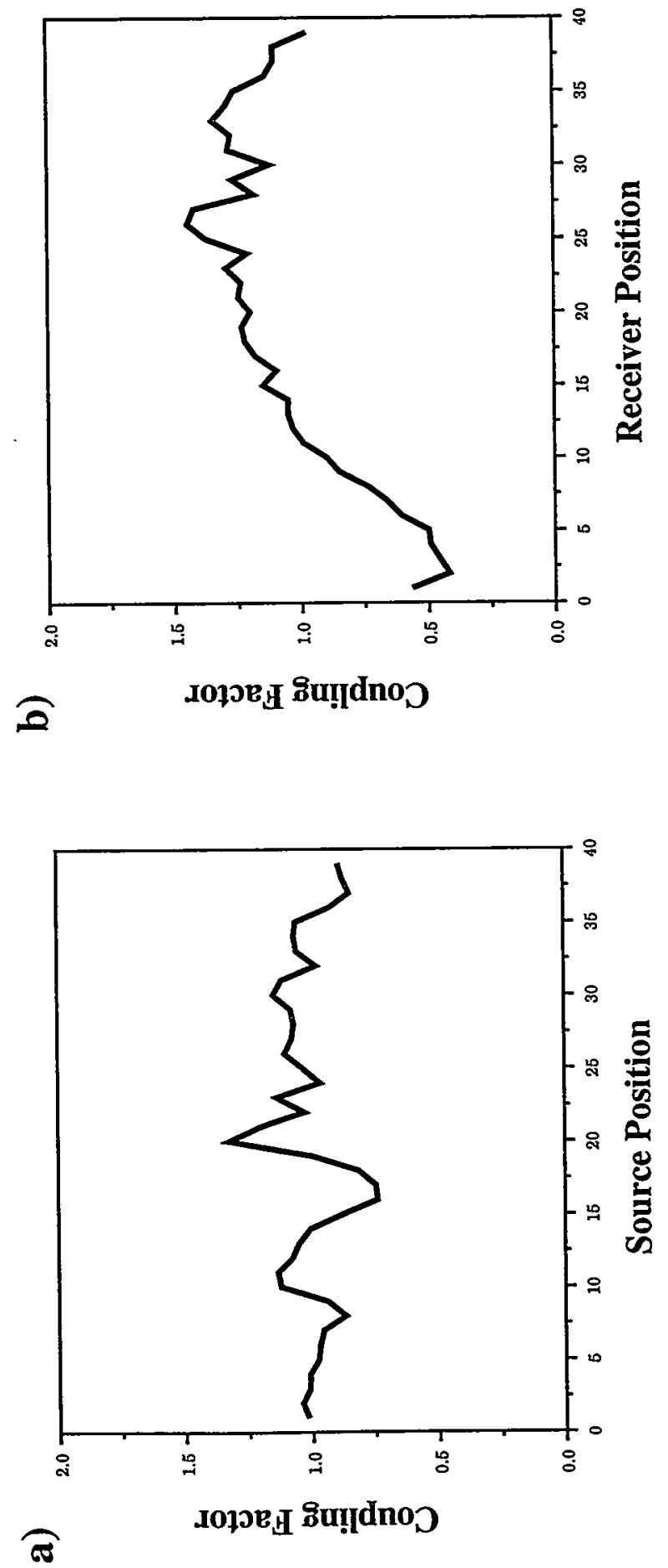


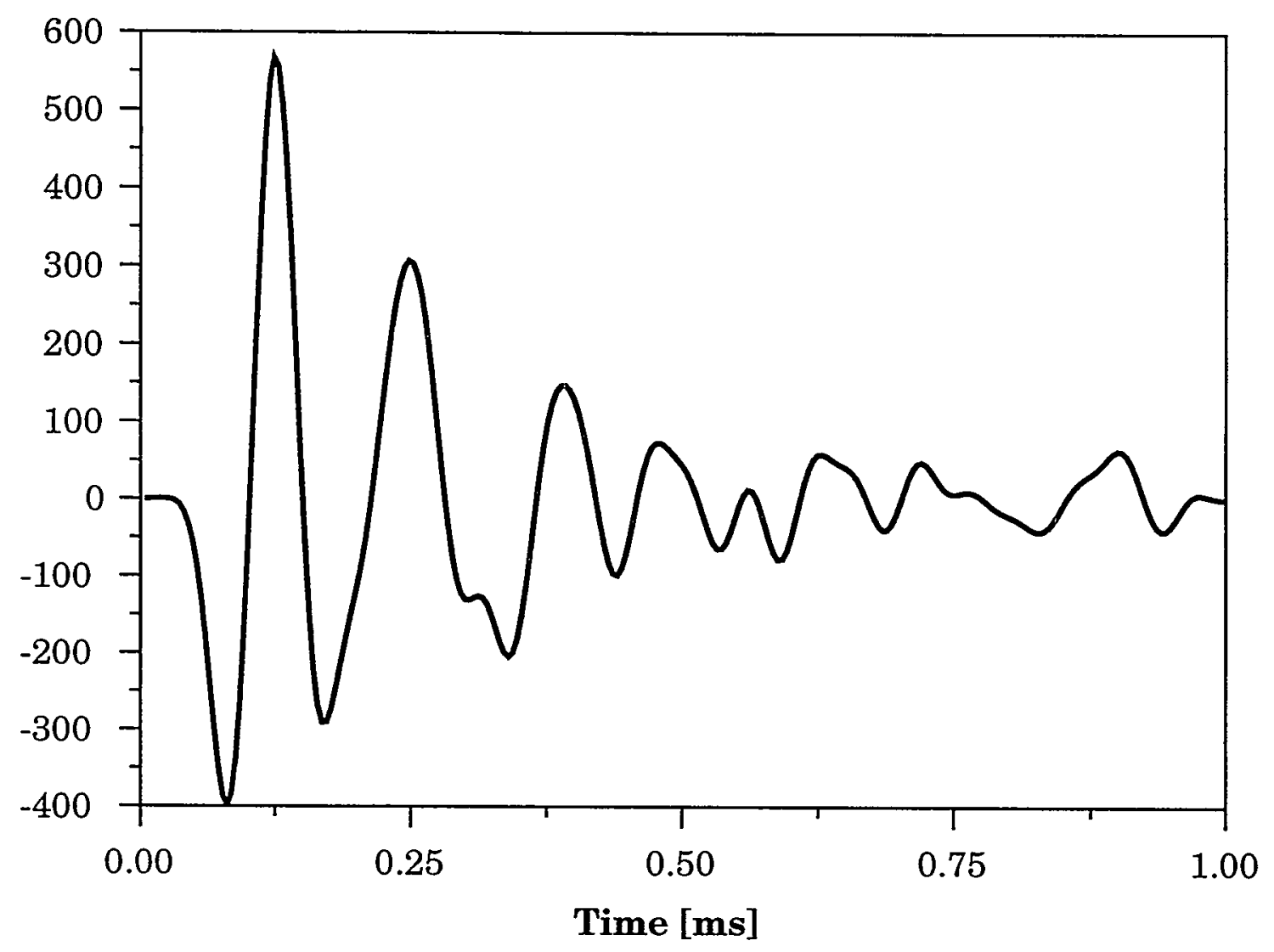

Figure 4 
罯
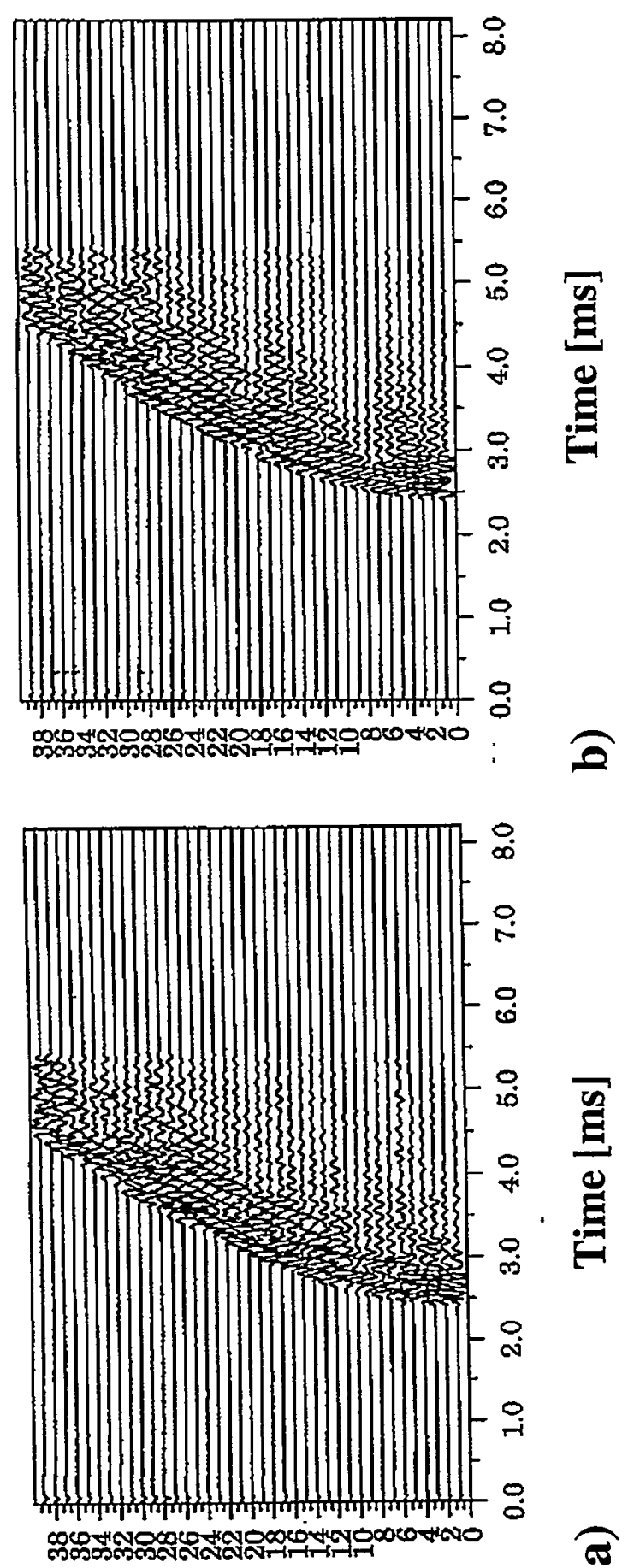


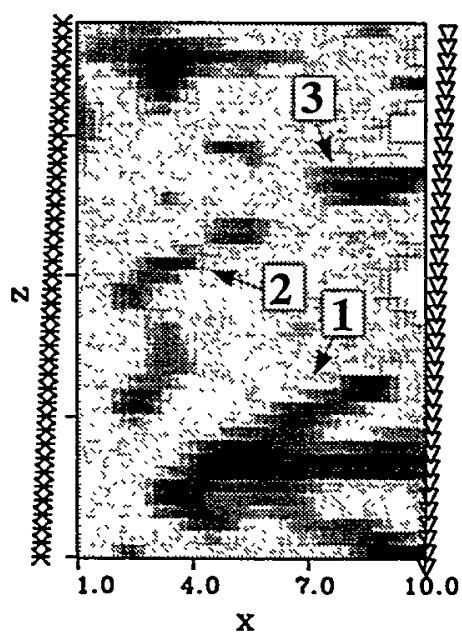

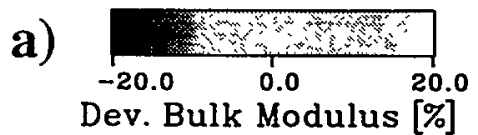

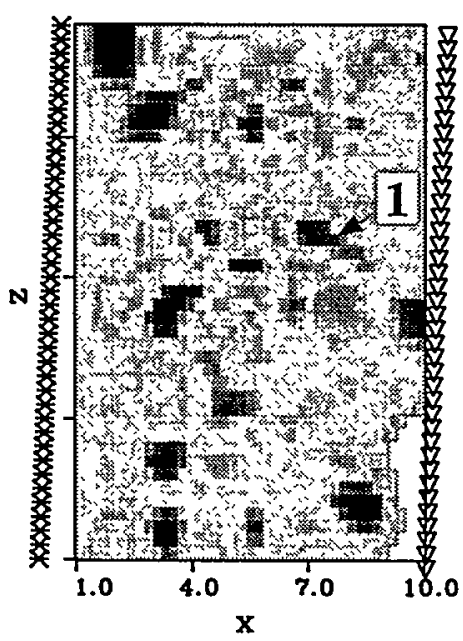

b) $\frac{1.0}{-9.0 \quad 0.0 \quad 980}$
Dev. Shear Modulus [\%]

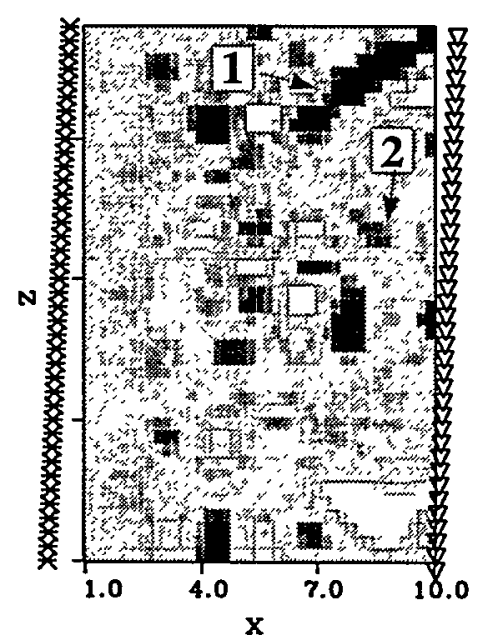

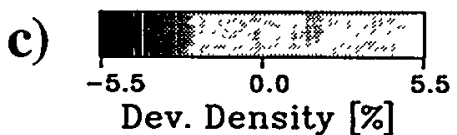




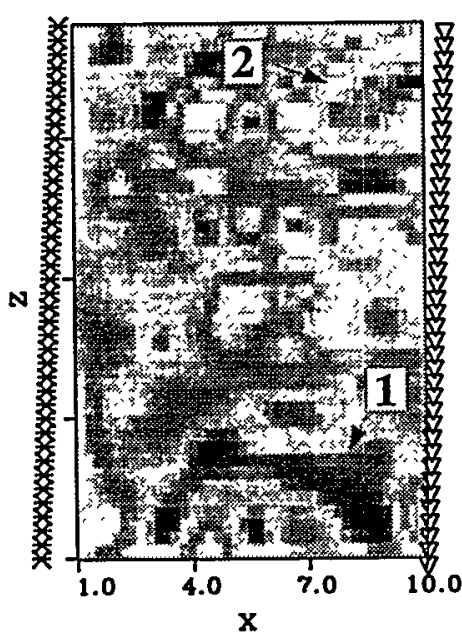

a) $\begin{array}{ccc} & & \\ -6.0 & 0.0 & 8.0\end{array}$ Dev. P Wave Velocity [\%]

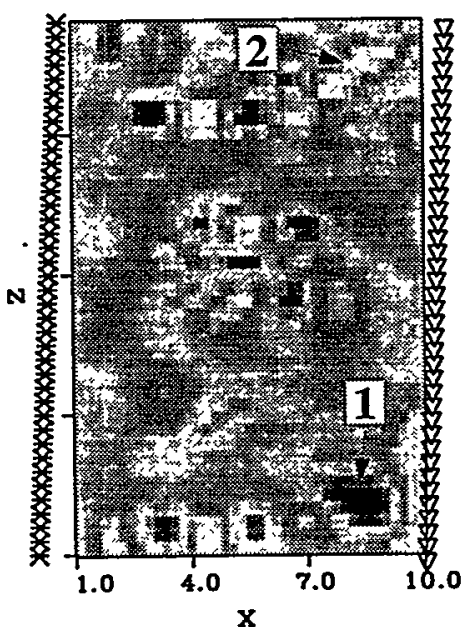

b) $\frac{}{-8.0} \quad 0.0 \quad 8.0$
Dev. S Wave Velocity [\%]

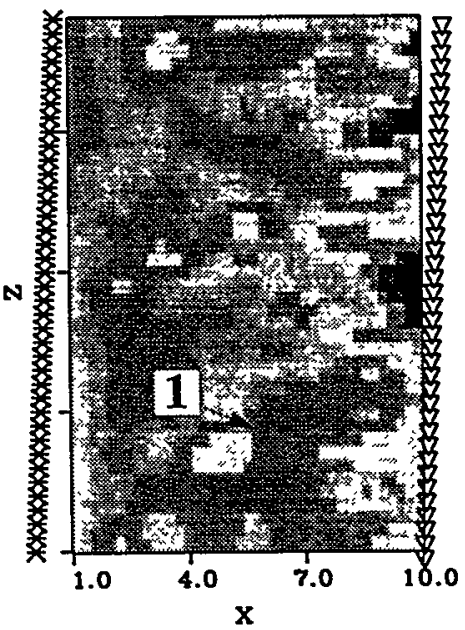

c) $\frac{1}{0.55 \quad 0.60}$ 


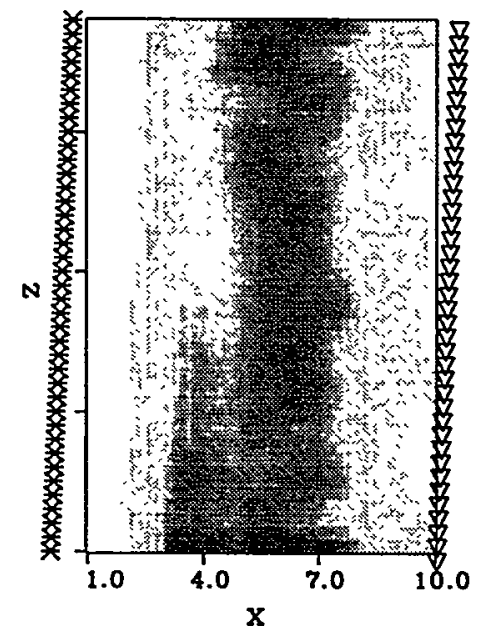

a) $\frac{1}{0.00 .2 \quad 0.4 \quad 0.6 \quad 0.81 .0}$

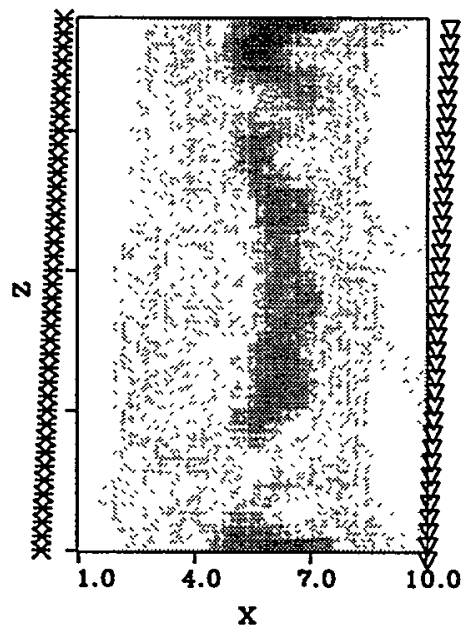

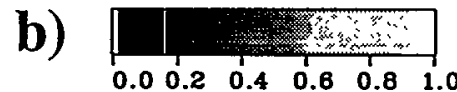
Resolution Shear Modulus
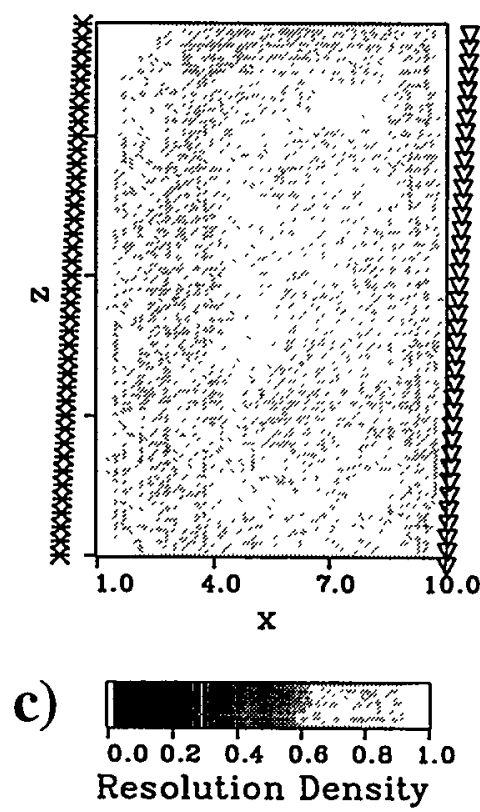

Figure 8 

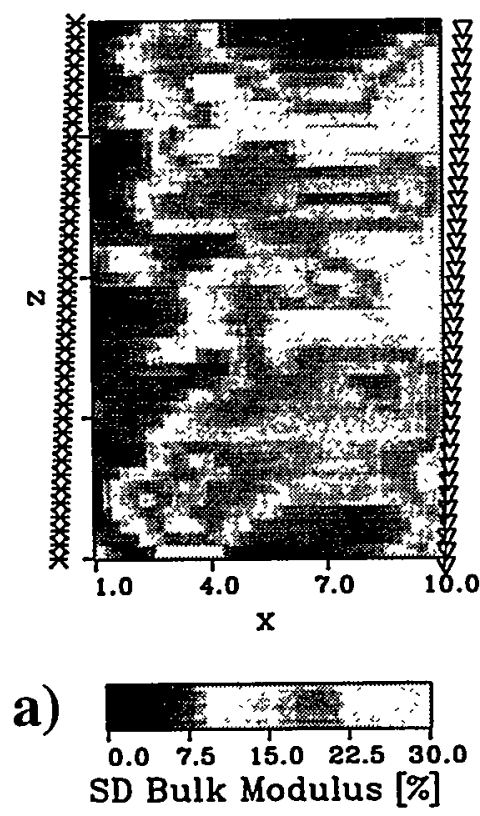

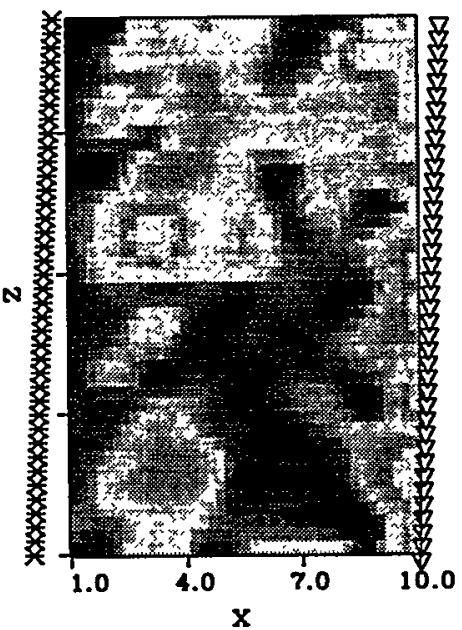

b)

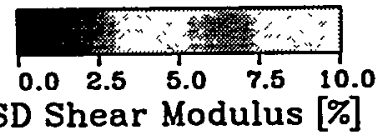

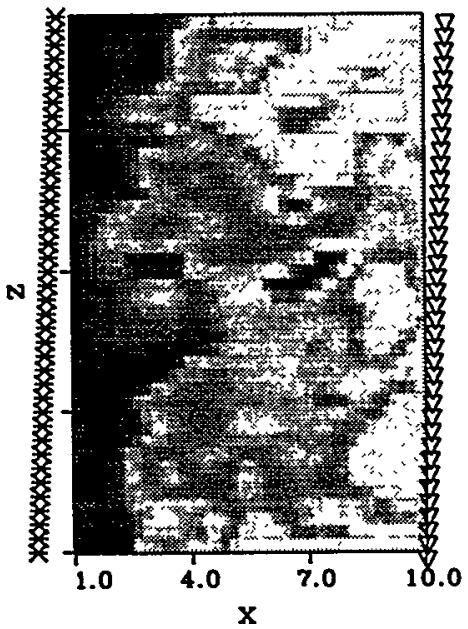

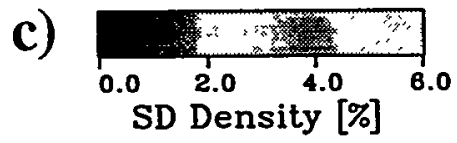

Figure 9 